\title{
Accounting for User Familiarity in User Interfaces
}

\author{
C. A. D'H Gough, R. Green, M. Billinghurst \\ HITLabNZ, University of Canterbury, New Zealand
}

Email: Christiaan.gough@hitlabnz.org

\begin{abstract}
Previous work discussed a model of cognitive distance with the novel concepts of "tech bias", "velocity" and "inertia". This paper examines the human aspects of the model by seeking to verify the expected user familiarity behaviour.

It describes a pilot study that suggests the model presented allows for a very high degree of confidence in predicting the effect a user's familiarity with a problem domain and specific implementation will have on their perception of the directness of the user interface, allowing for greater insight into the construction of optimally effective novel Augmented Reality interfaces.
\end{abstract}

Keywords: LaTeX style, conference paper, New Zealand conference

\section{Introduction}

Direct Manipulation is an approach to designing user interfaces, which forms the basis of Graphical User Interfaces.

A good understanding of how Direct Manipulation works is essential in engineering optimal user interfaces; especially in cases such as Augmented Reality, where the interfaces are often novel and highly unusual. A model allowing the prediction of the effectiveness of such novel interfaces prior to construction may save much time in their construction and subjective evaluation.

A previous paper ${ }^{1}$ presented a model of the relationship between the user and computer in a Direct Manipulation interface. This model related cognitive distance with user familiarity and the novel concepts of "tech bias", "velocity" and "inertia". This model may be used to compare user interfaces and explain or predict differences in the degree of "directness" or "distance" perceived by the user. The model defines the difference between perceived distance and directness as being "User Factors" primarily that of "User Familiarity".

In this paper these "Human Factors" are examined more closely, and studies intended to verify the exact effect of these factors are discussed.

\section{The model}

The model describes 2 key indices - the index of directness and the index of distance. The difference between the index of distance and directness is that of the human factors that contribute to a user's perception of how direct a user interface is.

\subsection{Index of Distance}

The first of these indexes is the Index of Distance $(S)$, which may be used to predict the distance a proposed user interface may present.

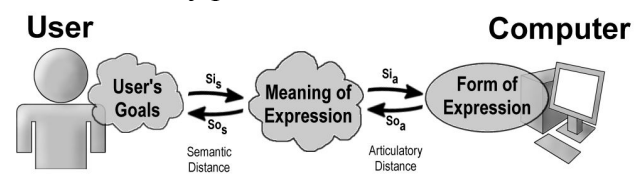

Cognitive Distance ${ }^{3}$ is a measure of the gulfs of execution and evaluation - the conceptual gap between the user's ideas and intentions, and the way in which they are expressed to, or represented by, the system.

Tech bias $(T:(0<T<1))$ is defined as "a measure of how well a given device succeeds in the role for which it is intended" 1 .

The index of distance scales the cognitive distance of the input and output channels by the Tech Bias of those channels respectively, as shown in equation (1):

$$
S=\left(\frac{S_{i s}+S_{i a}}{T_{i}}\right)+\left(\frac{S_{o s}+S_{o a}}{T_{o}}\right)
$$

Mature technologies - such as CRT and LCD displays, mice and keyboards are effective at providing their intended experience and, as such, tend to have a high tech bias. Conversely, less commonplace technologies usually have a relatively low tech bias.

In most cases the primary aim of developing an interface is to minimise distance irrespective of user experience, due to the variability of a large user base. 
The index of distance is therefore useful for comparing or considering user interfaces in terms that ignore the user factors, such as in the case of engineering a UI for mass-market acceptance.

\subsection{Index of Directness}

The sensation, as perceived by a user, of increased usability and interactivity provided by a good DM user interface is known as "directness".

The components of directness are those of the cognitive "distance" between the user and the computer $(S)$, and certain user-related factors $(U)$.

The Index of Directness $(D)$ describes how direct a given user perceives a given implementation of a given user interface to be. It is computed by scaling the index of distance by user factors $(U:(0<U<1))$ :

$$
D=U \times S
$$

These user factors $(U)$ were previously defined as familiarity with the user interface $(F:(0<F<1))$, yielding the following complete model:

$$
D=\frac{1}{F} \times\left(\left(\frac{S_{i s}+S_{i a}}{T_{i}}\right)+\left(\frac{S_{o s}+S_{o a}}{T_{o}}\right)\right)
$$

The Index of Directness is an important measure when dealing with a specific, specialised user scenario; where the overall perceived directness might be more relevant than the cognitive distance alone.

\subsection{Application}

The minimum attainable distance of a given UI is determined by the semantic and articulatory components of cognitive distance of the input and output channels, and the degree to which it is possible to achieve this theoretical minimum is governed by the user factors and tech bias of the hardware used.

This paper uses two layers of interaction - semantic and articulatory, but other common configurations could be used ${ }^{5,6,7}$.

Due to the inherent difficulties of deriving meaningful values for any of the coefficients used in the model, any evaluation of indices using this model should be used relatively rather than absolutely.

For example, it should not be assumed that an index of directness computed for one case may necessarily be compared directly with another, unless care were taken to use the same scales, assumptions and methodology in both cases.

\subsection{Velocity of Mixed Interfaces}

An interesting observation may be made in the case of applications where the user is exposed to "mixed distance interfaces", where various elements of the interface have differing distances.
A good example is that of a recording studio application, where a part is implemented tangibly as a "mixing desk" and a part is implemented via a traditional GUI, mouse and keyboard.

Such mixed-distance interfaces are a sensible approach to improving directness, as they allow a commonly used subset of tasks or operations to have a lessened cognitive distance without sacrificing the flexibility of a more traditional user interface for the less common tasks.

In such cases, it is useful to consider the change of distance that the user must overcome when switching focus between the interface elements. Such variations in distance within an interface can be described as "velocity".

By taking a weighted average of the Index of Distance for each of the interface types, we can derive a single overall Index of Distance and Index of Directness for the whole interface. This in turn means the theoretically optimal "blend" of interface types can be determined using linear programming.



Figure 1: A typical recording studio application represents a good mixed-distance user interface.

\subsection{Inertia}

If a user interface is significantly altered in order to improve distance, it must be determined if the gains in directness due to decreased distance are greater than the loss of directness caused by the decreased user familiarity. A small improvement in the distance of a system used by very expert users may not be enough to counter the expertise lost in changing the interface, resulting in a net loss of perceived directness to the user.

Thus, any reductions of distance in an existing user interface must be large enough to overcome the "inertia" of the users' experience if it is to be a worthwhile improvement without requiring relearning by the users.

For example, air traffic controllers spend a long time attaining expertise in using their systems. Because these systems are complex and because the safety of 
hundreds of lives relies on their effective use, there is much research on improving the user interfaces in order to reduce distance. It would be possible to engineer a new interface that greatly reduced distance using the Index of Distance; but in doing so, much of the acquired directness of the system by the controller may be lost.

In this case the index of directness should be used instead, in order to assess the improvements in light of the inertia of the controller using the system.

It is possible to argue that the primary focus should always be that of directness, as new systems may be re-learned and thus, with time, a new expertise may be joined with the decreased distance to achieve the most optimal possible usability. But consider that in some cases the user may have so much inertia that it is almost impossible to overcome.

For example, surgeons are provided important information via auditory cues during an operation, such as heart rate. Surgeons become so expert at using this system that their use of the interface is almost completely subconscious.

If the interface were re-engineered in such a way that this information was no longer provided, it could result in life-threatening performance decreases for the surgeon that are unable to be re-learned. Any replacement would in essence be a substitute, rather than a replacement, for the auditory approach.

\subsection{User Factors}

Previous work suggested that the user's sense of directness will be inversely proportional to their level of experience ${ }^{2,3}$ with the system because, as users become familiar with the interface, less cognitive effort is required to express their desires ${ }^{3}$.

The user factors that differentiate the index of distance from the index of directness were therefore previously expressed ${ }^{1}$ as the reciprocal of user familiarity:

$$
U=\frac{1}{F}
$$

\section{New Model}

This paper proposes an expanded definition of the user factors. It was reasoned that the user's familiarity with the problem domain of the application would be equal in effect to that of familiarity of the implementation of the application used - all other factors held constant - when determining the user's perceived directness with a given application.

The value of $U$ was therefore updated to take the following form:

$$
U=\frac{2}{F_{d}+F_{i}}
$$

where $F_{d}$ represents the familiarity of the user with the problem domain, and $F_{i}$ represents the familiarity of the user with the specific application in question.

\section{Pilot study}

A pilot study was performed to gain insight into the validity of this model. Participants were provided with a URL of a website containing a questionnaire.

Participants were able to $\log$ in to this website and answer a series of questions regarding their degree of experience with, and perception of, various implementations of operating systems and file operation environments. Several of these questions gave insight into the participant's experience with 4 specific operating systems - Windows XP, Mac OSX, Linux and Command Line Interfaces such as DOS.

Table 1: Questions asked in the pilot study. These questions were presented to the participant 4 times with a different OS replacing "X".

\begin{tabular}{|l|l|}
\hline 1 & $\begin{array}{l}\text { How familiar are you with X? [1 = very } \\
\text { unfamiliar, 5 = very familiar] }\end{array}$ \\
\hline 2 & $\begin{array}{l}\text { How would you rate your mastery of X? [1 = } \\
\text { not good, 5 = very good] }\end{array}$ \\
\hline 3 & $\begin{array}{l}\text { How competant do you feel in performing tasks } \\
\text { with X? [1 = very incompetant, 5 = very } \\
\text { competant] }\end{array}$ \\
\hline 4 & $\begin{array}{l}\text { How much do you enjoy performing tasks with } \\
\text { X? [1 = not very much, 5 = very much] }\end{array}$ \\
\hline 5 & $\begin{array}{l}\text { How confident are you when using X to perform } \\
\text { tasks? [1 = very unconfident, 5 = very } \\
\text { confident] }\end{array}$ \\
\hline 6 & $\begin{array}{l}\text { If you had to give an overall rating of X, what } \\
\text { would it be? [1 = very bad, 5 = very good] }\end{array}$ \\
\hline 7 & $\begin{array}{l}\text { How easy do you feel it was to learn to use X? } \\
{[1=\text { very difficult, 5 = very easy] }}\end{array}$ \\
\hline 8 & $\begin{array}{l}\text { How easy do you feel it is to learn new features } \\
\text { of X? [1 = very difficult, 5 = very easy] }\end{array}$ \\
\hline 9 & $\begin{array}{l}\text { How confident are you in your ability to retain } \\
\text { your current mastery of X? [1 = very } \\
\text { unconfident, 5 = very confident] }\end{array}$ \\
\hline 10 & $\begin{array}{l}\text { How eager would you be to demonstrate the use } \\
\text { of X or train novices in using X? [1 = not very } \\
\text { eager, 5 = very eager] }\end{array}$ \\
\hline 11 & $\begin{array}{l}\text { How much do you want to explore the more } \\
\text { powerful aspects of X? [1 = not at all, 5 = very } \\
\text { much] }\end{array}$ \\
\hline 12 & $\begin{array}{l}\text { How easily do you feel you can achieve a given } \\
\text { task using X? [1 = not very easily, 5 = very } \\
\text { easily] }\end{array}$ \\
\hline 13 & $\begin{array}{l}\text { How much do you feel that X is a tool or } \\
\text { extension of yourself, rather than part of the task } \\
\text { to be achieved? [1 = not at all, 5 = very much] }\end{array}$ \\
\hline
\end{tabular}


The remaining questions were intended to gather an appreciation of the participant's perceived directness of the operating systems, based on the list of proposed benefits of a good DM interface described by Shneiderman. All questions were to be answered using a Likert Scale of 1-5.

The questions were duplicated exactly for each operating system so that, in effect, each participant was completing the same questionnaire 4 times for different Operating Systems.

\section{Results}

The results from 22 participants were processed in such a way that 88 samples were obtained, where each sample represented a set of results of one participant's rating of their experience and perceived directness of an individual Operating System. Each of these results are represented on figures 2,3 and 4 as a single diamond.

The results of the questions pertaining to the participant's familiarity with a given OS were averaged for each sample to obtain a value for their $F_{i}$ for that OS, and the remaining questions of that sample were averaged to represent the participant's perceived Directness $(D)$ for that sample.

The resulting correlation between $F_{i}$ and measured $D$ gave a good correlation $\left(R=0.863, R^{2}=0.745\right)$ (fig 2 )

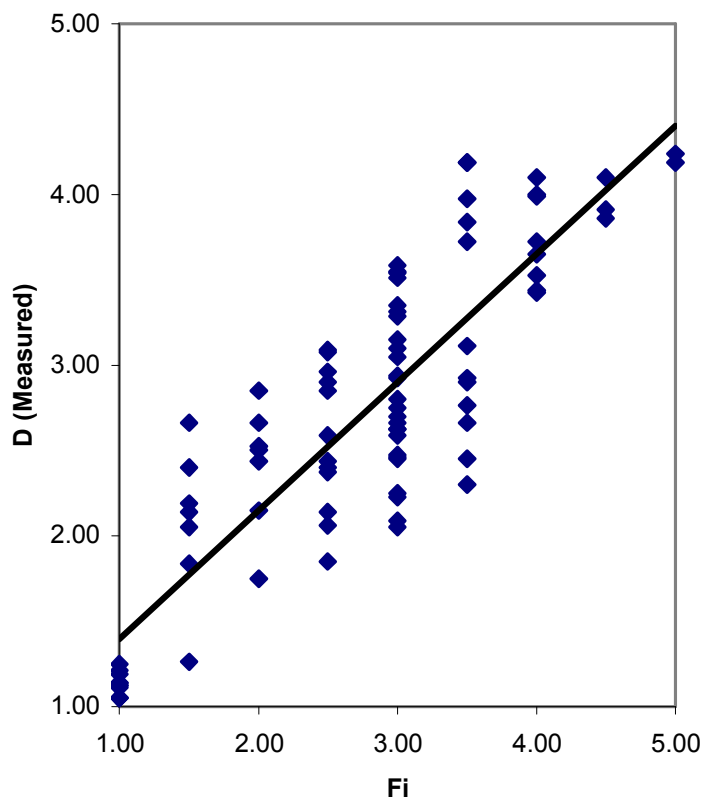

Figure 2: Fi versus Measured D

The $F_{d}$ of each participant was then computed by averaging the $F_{i}$ of each of the 4 OS samples for that participant. Plotting the correlation between each participant's $F_{d}$ and the $D$ for each of their samples gave a low correlation $\left(R=0.448, R^{2}=0.201\right)$ (fig. 3$)$.

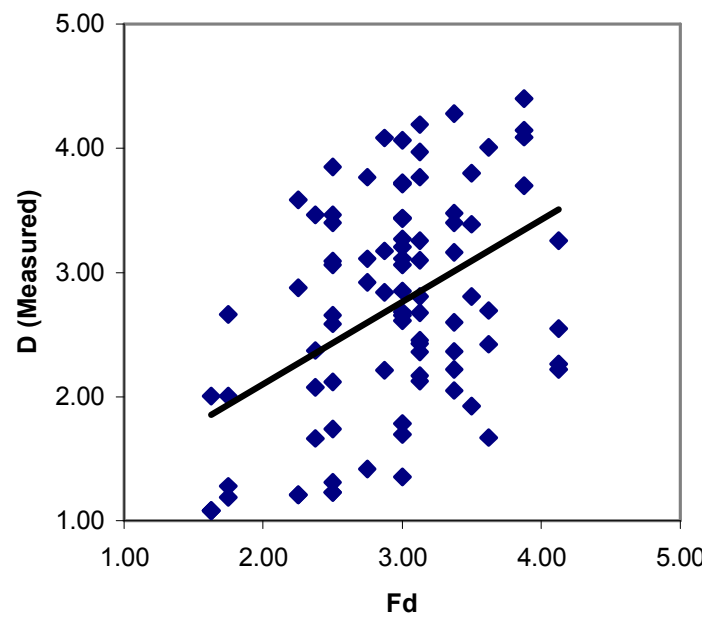

Figure 3: Fd versus Measured D

Finally, the $U$ for each sample of each participant was computed using the method proposed by this paper by averaging the $F_{d}$ and $F_{i}$ for each sample.

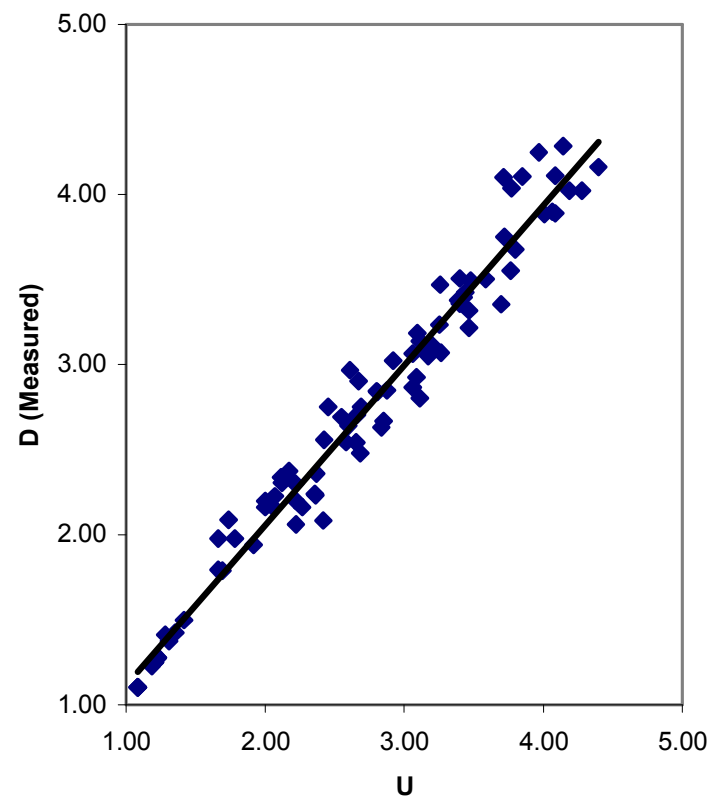

Figure 4: U versus Measured D

The resulting $U$ for each sample of each participant was very highly correlated $\left(R=0.983, R^{2}=0.967\right)$ (fig. 4). 


\section{Conclusion}

The results of this pilot study suggest that a given user's perception of directness of a given user interface may be accurately predicted using the model described in this paper.

This model is significantly more accurate than the traditional approach of simply assuming perceived directness will be proportional to their familiarity with the interface alone.

The pilot study suggests that the model described may allow confidence of over $90 \%$, although this needs to be verified with more rigorous experimentation.

A good understanding of how this effect works is essential in engineering optimal user interfaces; especially in cases such as Augmented Reality, where the interfaces are often novel and highly unusual. A model allowing the prediction of the effectiveness of such novel interfaces prior to construction may save much time in their construction and subjective evaluation.

\section{Future Work}

This study was an initial stage in verifying and examining the theories described by Gough et al.

More exhaustive studies are currently being carried out to provide greater insight into the results indicated by the pilot study described in this paper. These experiments are as follows:

\subsection{Experiment 1: File operations}

This experiment is to be a more exhaustive and rigorous version of the pilot study described in this paper.

The problem domain will be restricted to that of file operations alone, rather than more general usage of Operating Systems.

Participants will be asked to perform a variety of file operation tasks using a variety of approaches, including some approaches that will be customimplemented so as to allow greater focus on the relationship between $\mathrm{Fd}$ and Fi.

The purpose of this experiment is to gain insight into the role of user familiarity with a problem domain and implementation, and to replicate the results of this paper with more accuracy and rigour.

\subsection{Experiment 2: Creative content}

Participants will be asked to "mix" a song based on content provided to them.

Participants will have varying experience in the use of computers, audio editing and mixing, and in performing and creating music.
Mixing will take place on a mixing desk alone, a computer alone, and a mixed-distance interface consisting of an automated mixing desk coupled with an interoperable software environment on a connected computer.

Once again, the purpose of the experiment is to gain insight into the role of user familiarity with a problem domain and implementation, and to replicate the results of this paper with more accuracy and rigour.

There will, however, be additional scope to gain insight into the effect of the effect of mixed-distance user interfaces on perceived Directness, and specifically the interrelation between the indices of distance and directness under a mixed-distance situation.

\subsection{Experiment 3: DB Query}

Participants will be asked to perform a series database search queries. The queries will be via traditional user interfaces such as a web-based search engine, an SQL command string and a form-based Access GUI.

Participants will also be provided with several new graphical and tangible approaches based on both new existing metaphors.

The benefit of this research will once again be primarily that of verification of the existing model, but will also allow unique insight into other potential factors unaccounted for at present, as well as into the interplay of the indices of directness and distance.

\subsection{Experiment 4: IDE Usage}

The final experiment will require users to perform a series of common tasks using development environments. The questions listed in table (1) will be asked of the participants, and correlated in the same way as the pilot study and previous experiments.

\section{References}

[1] Gough, C. Green, R. Billinghurst, M., "Better Realising Direct Manipulation", Image and Vision Computing New Zealand 2005, pp. 455-460.

[2] Shneiderman, B., "The Future of Interactive Systems and the Emergence of Direct Manipulation", Behaviour and Information Technology 1982, v.1 n.3, pp. 237-256

[3] Hutchins, E. Hollan, J. Norman, D., "Direct Manipulation Interfaces", Human-Computer Interaction, Volume 1, 1985. pp. 311-338

[4] Frohlich, D, "The history and future of direct manipulation" Behaviour \& Information Technology 12, 6, 1993. pp. 315-329.

[5] Neilsen, J, “A Layered Interaction Analysis of Direct Manipulation”, 1992 
[6] Hix, D. and Hartson, H., "Developing User Interfaces". John Wiley \& Sons, Inc, 1993

[7] Taylor, M., "Layered protocol for computerhuman dialogue", I: Principles. International Journal of Man-Machine Studies, 28, 1988. pp. $175-218$

[8] Antifakos, S. "Improving Interaction with Context-Aware Systems", Selected Readings in Vision and Graphics, volume 35, 2005

[9] Ishii, H., Kobayashi, M., Grudin, J. "Integration of Interpersonal Space and Shared Workspace: ClearBoard Design and Experiments," ACM Transactions on Information Systems (TOIS), ACM, Vol. 11, 1993

[10] Nelles, C. "Graphical vs Tangible User Interface", unpublished $\mathrm{PhD}$ thesis, eingereicht am FachochschulDiplomstudiengang, Medientechnik und Design, Hagenburg, 2005 